Document downloaded from:

http://hdl.handle.net/10251/178897

This paper must be cited as:

Jordán, J.; Valero Cubas, S.; Turró, C.; Botti Navarro, VJ. (2020). Recommending Learning Videos for MOOCs and Flipped Classrooms. Springer. 146-157. https://doi.org/10.1007/9783-030-49778-1_12

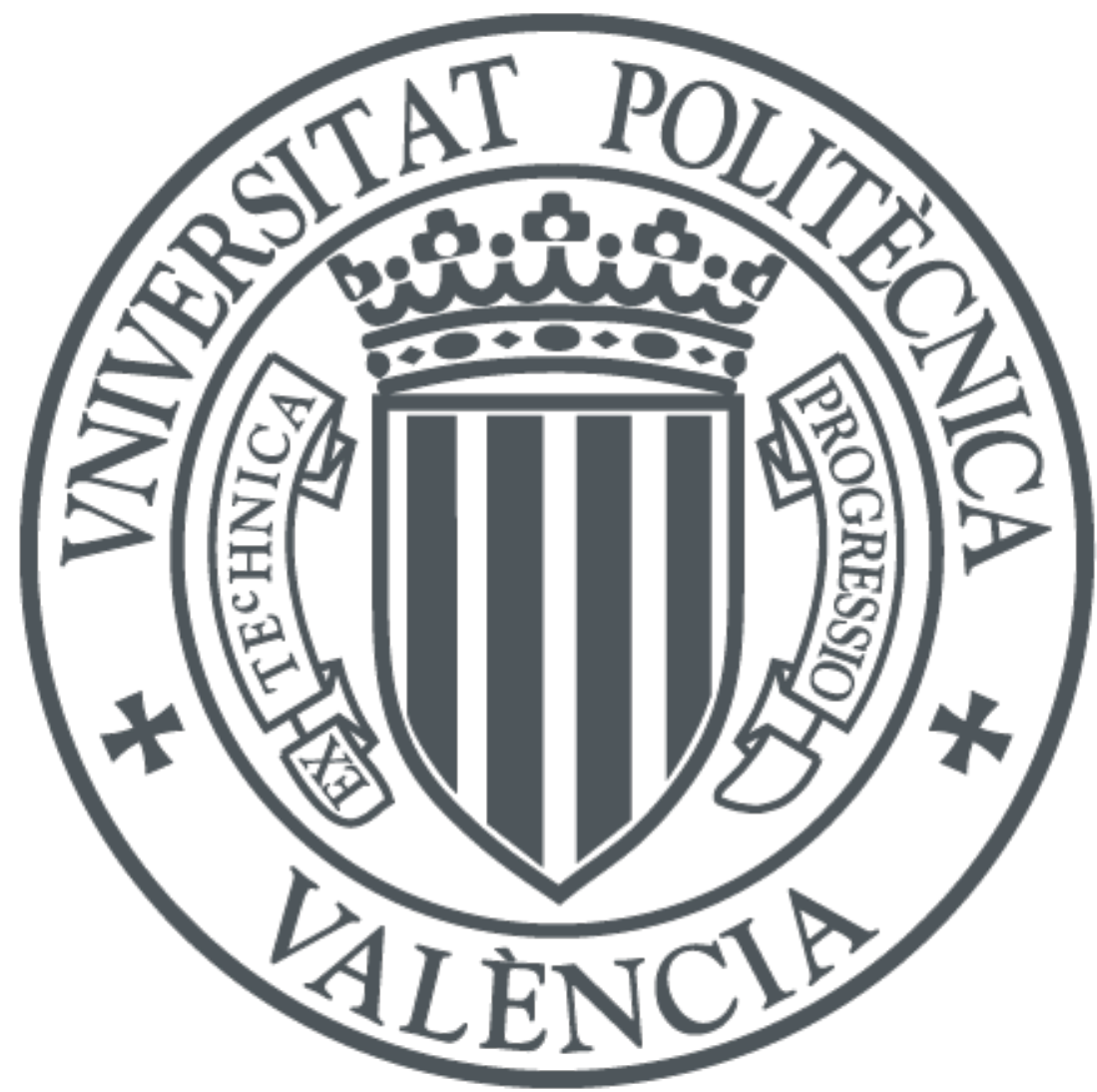

The final publication is available at

https://doi.org/10.1007/978-3-030-49778-1_12

Copyright Springer

Additional Information 


\title{
Recommending learning videos for MOOCs and flipped classrooms
}

\author{
Jaume Jordán ${ }^{1[0000-0003-0400-9136]}$, Soledad Valero ${ }^{1[0000-0003-4565-326 X]}$, \\ Carlos Turró 2 [0000-0002-3840-9405], and Vicent Botti1 [0000-0002-6507-2756] \\ 1 Valencian Research Institute for Artificial Intelligence (VRAIN), Universitat \\ Politècnica de València, Camino de Vera s/n, 46022 Valencia, Spain. \\ $\{$ jjordan, svalero, vbotti\}@dsic.upv.es \\ http://vrain.upv.es/ \\ 2 Área de Sistemas de Información y Comunicaciones. Universitat Politècnica de \\ València, Camino de Vera s/n, 46022 Valencia, Spain. \\ turro@cc.upv.es
}

\begin{abstract}
New teaching approaches are emerging in higher education, such as flipped classrooms. In addition, academic institutions are offering new types of training like Massive Online Open Courses. Both of these new ways of education require high-quality learning objects for their success, with learning videos being the most common to provide theoretical concepts. This paper describes a hybrid learning recommender system based on content-based techniques, which is able to recommend useful videos to learners and teachers from a learning video repository. This hybrid technique has been successfully applied to a real scenario such as the central video repository of the Universitat Politècnica de València.
\end{abstract}

Keywords: Learning Recommender System · Learning Object · Learning Videos · Content-Based

\section{Introduction}

The profile of students has changed in the last decade. They demand new ways of learning, better adapted to their way of life and moving away from classical teaching.Academic institutions must increasingly adapt their teaching methodology to the new required ways, taking into account the opportunities offered by the global world [10]23. In this way, there has been a great increase in the supply of Massive Online Open Courses (MOOCs) by academic institutions, as well as in the number of students who opt for this type of training [5. This kind of courses are mainly based on learning objects (LOs). As IEEE propose, a LO is "any entity, digital or non-digital, which can be used, re-used or referenced during technology supported learning" 8 . Thus, MOOCs use different kinds of LOs, being videos the common ones to provide theoretical concepts. In addition, new teaching approaches are emerging in higher education, such as flipped teaching [12, 15, 19, in which the theoretical contents are studied at home by the students, while the face-to-face sessions are eminently practical, 
where the knowledge acquired through problem solving is put into practice. To this end, the teacher indicates to the students which LO they should work on at home before the next face-to-face session. Thus, students are proposed to acquire the theoretical concepts not only through books or specialized articles, but also through audio-visual material.

Universitat Politècnica de València ${ }^{3}$ (UPV) is a Spanish Public university which offers undergraduate degrees, dual degrees, masters and doctoral programs. UPV has more than 28000 students. In the last decade, UPV has been promoting new pedagogical methodologies in their degrees, such as flipped teaching 21. It has also made a great effort in developing MOOCs within the edX platform 4 , with more than 2 million enrollments and having three courses in Class Central all time top 100 MOOC 5 .

Students in blended and flipped classroom environments need access to a variety of resources to understand the theoretical concepts required. In order to facilitate this task, UPV has a long time project of digital resources aiming to produce video content as LOs 22. This video content is managed in the University central video repository, called mediaUPV ${ }^{6}$. This portal is also used for MOOCs and other related projects.

mediaUPV allows UPV teachers to upload and manage video content for students. Students access mediaUPV usually through recommendations of their teachers through the Learning Management System (LMS), but they also access the video portal on their own, and browse through the contents. A relevant feature of mediaUPV against other alternatives (e.g. YouTube) is that videos have been developed by teachers from the institution, so there is a guarantee of the content quality.

While mediaUPV is very important to the institution, its size constitutes a growing problem, because it is increasingly difficult to find the most relevant content for both students to view and teachers to prescribe. Thus, UPV decided to look into a new Learning Recommendation System that could recommend relevant and related videos. This paper describes the proposal of the recommender engine designed and developed, and also its results in production.

This article is structured as follows. In Section 2 related works are described. Following, Section 3 specifies a description about the LOs to recommend and the potential users of the system. In Section 4 the proposed recommender system is explained. Then, Section 5 shows the experimental results of the proposed recommender in the mediaUPV portal. Finally, Section 6 draws the conclusions and future work of this paper.

\footnotetext{
3 http://www.upv.es

4 https://www.edx.org/

5 https://www.classcentral.com/report/top-moocs-2019-edition/

6 https://media.upv.es/
} 


\section{Related Work}

Learning Recommender Systems (LRS) should assist learners in discovering relevant LO than keep them motivated and enable them to complete their learning activities 9. Most of the LRS adopt the same techniques than regular recommender systems [3] [7] [14, such as: content-based, in which recommendations are determined considering user profiles and content analysis of the learning objects already visited by the user; collaborative filtering, in which recommendations are based on the choices of other similar user profiles; knowledge-based, in which it is inferred whether a LO satisfies a particular learning need of the user to recommend it; and hybrid, in which recommendations are computed by combining more than one of the above techniques.

In recent years, different approaches have been proposed in order to improve the efficiency and accuracy of the recommendations and retrieval of useful LOs. In this way, in [2], authors provide new metrics for applying collaborative filtering in a learning domain, so users with better academic results have greater weight in the calculation of the recommendations. However, the experiments did not carried out in a learning environment. Other proposals focus on recommending to students those LOs that can be most useful to them, providing solid arguments. This is the case of [14, which combine content-based, collaborative and knowledge-based recommenders using an argumentation-based module to recommend LOs inside a LMS. In this case, information on student profiles and learning styles is also available. An item-based collaborative filtering method is combined with a sequential pattern mining algorithm to recommend LOs to learners in [4. In this case, LOs are ranked by the students and it is also possible to obtain the browsing sequences made by them. In a similar way, [18, proposes a hybrid knowledge-based recommender system based on ontology and sequential pattern mining for recommendation of LO. Authors can adequately characterize learners and LOs using an ontology, since they have detailed information about them. In [6], the authors propose a method for recommending LOs to a group of individuals, building a unified learner profile which is used to recommend using a collaborative filtering approach.

Besides, other works have been done to improve the accuracy of the searches in mediaUPV. For example, in [17] a semi-supervised method is applied to cluster and classify the LOs of mediaUPV, obtaining specific keywords that represent each cluster. In [20, authors applied a custom approach for indexing and retrieving educational videos using their transcripts, which are available in mediaUPV. Videos are classified in different domains using the method described in [17. Also, they applied a Latent Dirichlet Allocation algorithm 1 to get a list of topics and their score. User queries are classified in one of the domains, recovering from that cluster those videos whose transcripts are the closest to the query.

As can be seen, most previous work on LRS adopts a hybrid strategy, seeking to harness the strength of each particular technique, overcoming its limitations by using them together. Furthermore, the different strategies that can be applied depend on the data available to describe LOs and users. In our case, a 
hybrid strategy will also be applied, combining content-based methods. On the other hand, previous experiences on the improvement of searches in the mediaUPV repository show us the usefulness of characterizing LOs using available transcripts and titles.

\section{Problem description}

mediaUPV portal started in 2011 and by the end of 2019 it holds 55600 different videos, mainly from STEM topics, with more than 10 million views. From this database, only 13232 are certified as high quality LOs. All these high quality videos have a transcript (with more than 100 characters), a title and an author, however the videos are not classified using any taxonomy and no keywords are associated to them. mediaUPV platform generates the transcripts of what is said in the videos using the poli[Trans] service 7 , an online platform for automated and assisted multilingual media subtitling offered by UPV. poli[Trans] service is based on transLectures-UPV Platform [11].

mediaUPV portal is used by students and trainers. The students are mainly formal students of the UPV, but it also receives many visits from anonymous users, who can register in some MOOC offered by the UPV. Moreover, mediaUPV is not connected to the LMS of the UPV, so even though the user is authenticated, it is not possible to know his student profile (e.g. enrolled subjects).

Therefore, the aim of this proposal is to be able to offer recommendations not only to authenticated users but also to anonymous users of the system. In addition, the system should be able to recommend not only students, but also teachers who are looking for suitable material for their students. In all cases, the aim is to be able to offer recommendations on learning videos (LOs) considered by the UPV to be of high quality.

\section{Learning Recommender System Proposal}

Our LRS is based on two different approaches. On the one hand, a recommender module based on the activity of the user identified in the system, the profilebased module. On the other hand, another recommendation module based on the content of the video being watched at the time, the item-based module. Thus, the computed recommendations are based on both methods, getting a hybrid recommendation system, so the user receives recommendations from videos similar to the one he is currently watching, but also from videos that may interest him due to his viewing history.

In our proposal, LOs/videos are characterized by their title and their transcript. This characterization is used for the calculation of the similarity between the LOs. As transcripts are in different languages, it is possible to recommend videos from different languages.

\footnotetext{
7 https://politrans.upv.es
} 
Because there is a large set of words in the transcript and title of the videos, it is necessary to have an algorithm that filters out the too common words, which do not serve to differentiate the content. Thus, it is possible to focus on the particular words in the entire collection, which serve to identify the content of a video. Therefore, the algorithm chosen is the well-known term frequency inverse document frequency (tf-idf) [16. In order to improve the performance of the tf-idf algorithm, also the stop words from the nltk Python ${ }^{8}$ package are used. Furthermore, some ad hoc words have been added to this package.

The item-based module takes only the information from the content of the videos, i.e., there is no information about the users. So, we can consider this module as a recommendation by item-item similarity to be used when a (maybe anonymous) user is watching a video. To do this, we take the characteristics (transcript and title) of each of the videos to calculate the item-item matrix with the tf-idf algorithm. Then, the cosine similarity of two instances of this itemitem matrix returns the similarity among the different videos. Cosine similarity calculates the similarity between two n-dimensional vectors by the angle between them in the vector space:

$$
\operatorname{cosine\_ sim}(\vec{p}, \vec{q})=\frac{\vec{p} \cdot \vec{q}}{|\vec{p}| *|\vec{q}|}
$$

The profile-based module considers the content information (the terms extracted by the tf-idf algorithm from the transcript and title) of the videos viewed by users. In this way, the recommendations to a user are made based on the similarity among the viewed videos of the user in mediaUPV. In this case, the similarity is calculated using the cosine profile-item matrix.

Finally, our hybrid recommendation system consists of the two modules described above, balancing the results of both by means of configurable weights, i.e., $w_{I B}$ for the item-based weight and $w_{P B}$ for the profile-based weight. In this way, the recommendations are made taking into account the intersection of the videos recommended by both modules and completing the rest of the recommendations with the corresponding videos of the modules according to their weight. Also, in cases where there is no user (anonymous) the $w_{P B}$ is set to 0. Likewise, when an authenticated user is not yet watching a video, the $w_{I B}$ is set to 0 .

\section{$5 \quad$ Experimental Results}

In this section, we explain the experiments carried out with the proposed LRS. On the one hand, we have made tests with data from the videos seen by the users, dividing this data into a training set and a test set (Section 5.1). On the other hand, we have taken the parameter configuration of the recommender system that has worked best in this first phase and applied it to recommend in production at the mediaUPV portal; these results are shown in Section 5.2 .

\footnotetext{
8 https://www.nltk.org/
} 


\subsection{LRS parameter setting}

The data set used in the setting up process is formed by of learning videos viewed by users during an academic year at the UPV, from September 2018 to July 2019. The training data set consists of the data from September 2018 to April 2019, while the testing data set consists of the data from May to July 2019, i.e., 8 months for training and 3 months for testing. The videos considered are those present on the platform until July 2019, after filtering them as described above. Thus, we try to simulate a real scenario, in which the training data represent the past activity of the users, while the test data is formed by the activity of the following 3 months ("future"). Therefore, any recommendation from the recommender that is among the videos that users have actually seen in the test set is considered a success.

We focus on the hybrid recommender engine since it brings together the efforts of the two recommender modules. The weights for each module are set to $w_{I B}=50 \%$ and $w_{P B}=50 \%$. Additionally, in these tests we only consider 5 recommendations since it is the number required by the mediaUPV portal.

We use the well-known precision and recall measures to evaluate the success of the recommender. Precision can be defined as the successful recommendations made (videos that have been viewed by the user in the test set) divided by the number of recommendations made: precision $=\frac{\text { success_recommendation }}{\text { recommendations_made }}$. Recall is defined as the successful recommendations made divided by the number of watched videos in the test set: recall $=\frac{\text { success_recommendation }}{\text { watched_videos }}$.

For our tests we considered a set of regular users (reg_users) that we define as those who have seen between 10 and 150 videos both in the training and test periods, having 1044 users in this set.

Setting transcript and title features. In this first test, we analyze the success of recommendations for the set of regular users considering different amount of features for the transcript and the title of the video to train the LRS, in order to establish the better amount of both. The graph in Figure 1a shows the precision and recall for different values of the number of features considered for the transcript, while the number of features for the title is kept at zero.

In general, precision and recall increase slightly as the value of the features for the transcript increases (from $7.2 \%$ to $7.7 \%$ for precision, and from $1.15 \%$ to $1.29 \%$ for recall), as would be expected when more information is available from the transcript. The best precision values are with 35000 and 45000 features for the transcript. However, the best recall value is in the case of 35000 transcript features. Therefore, the best configuration for the recommender would be to use 35000 transcript features, since this value achieves higher recall than any other and equals the precision obtained with 45000 transcript features. In addition, the computation of 35000 transcript features is computationally less expensive and, in particular, implies a lower memory cost. It should be noted that, in the experiment made with 35000 transcript features, the number of users who have been recommended successfully is 254 of 1044 , i.e., $24.33 \%$. 


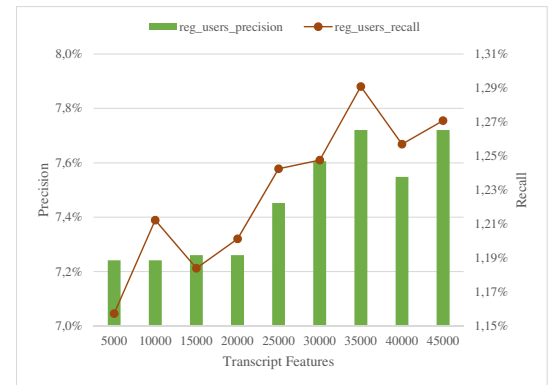

(a) Different amount of transcript features.

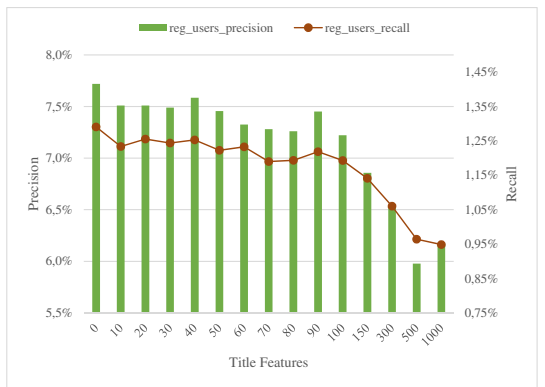

(b) Different amount of title features.

Fig. 1: Precision and recall analysis for the regular users set.

Figure $1 \mathrm{~b}$ shows the precision and recall for different values in the number of features considered for the title having the transcript features fixed to 35000 . The best values of precision and recall are obtained with the number of features of the title at 0 . In addition, both values decrease slightly and in a relatively uniform way as the number of title features increases. So, apparently it is better to skip the title features. However, it is interesting to analyze this considering the nature of the LOs of mediaUPV. In this way, we have analyzed the set of words that determines the tf-idf algorithm. As we mentioned before, the videos on the platform correspond mainly to university courses, so there is a set of terms that are certainly repetitive in the titles of the videos and do not provide any differentiating information with respect to their content. Among these terms, we find the following: \{'analysis', 'calculation', 'control', 'creation', 'data', 'design', 'exercise', 'engineering', 'introduction', 'management', 'mechanism', 'model', 'module', 'practical', 'practice', 'presentation', 'simulation', 'system', 'systems', 'theme', 'unit', 'virtual'\}.

Filtering title features. In order to increase the precision of the recommendations, we decided to filter the terms of the previous list from the titles of the videos by considering them as stop words. The results of applying this correction can be seen in the Figure 2a, which shows the precision and recall for 35000 transcript features and different amount of title features. In this case, it can be seen that the best global values of precision and recall are still obtained with 0 title features. However, it should be noted that with 10 title features, for the case where the specified title terms have been filtered out, the precision and recall almost reach this base case, slightly surpassing the case without filtering (with 10 title features). In addition, for values up to 30 title features, the precision and recall are better for the case with filtered terms. However, from 40 title features on, the effect of filtering is diluted and the results are generally slightly worse, and most of the cases slightly worse than the unfiltered case. 
Consequently, we can say that filtering has significantly improved the results but it is not enough to make the title relevant. Perhaps it would be necessary a still greater filtering of terms that we have not considered 'common' and that the tf-idf algorithm has not identified as such either. However, since we are considering 35000 transcript terms, the inclusion of 10 to 50 terms from the title can be considered irrelevant after the analysis. Furthermore, it can also be interpreted as a video being better characterized by its own transcript than by its title.

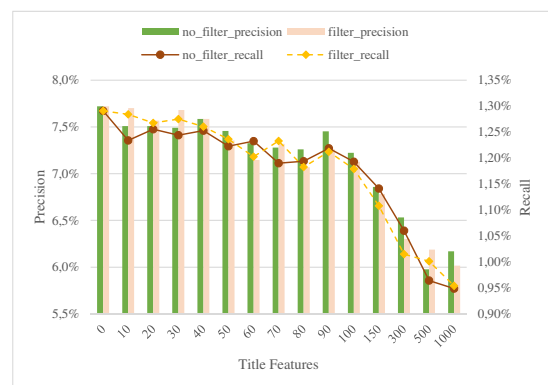

(a) Different amount of title features.

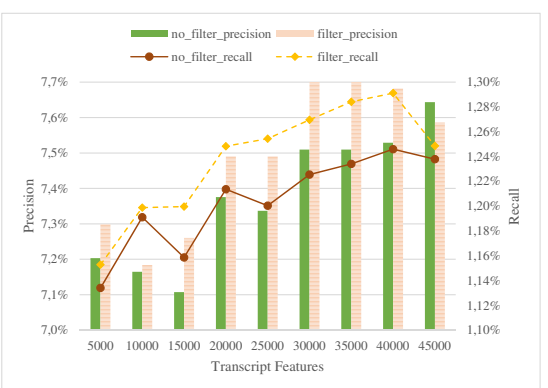

(b) Different amount of transcript features with 10 title features.

Fig. 2: Precision and recall comparison with and without filtering title terms.

Although we have already seen that it is better to skip the title in all cases, we will analyze in detail the difference between filtering the title terms and not filtering them for 10 title terms and different amount of transcript features. Figure $2 \mathrm{~b}$ shows this comparison (precision and recall) with and without filtering. Precision and recall are significantly better if filtering of title terms is performed in all cases, with the only exception of 45000 transcript features where precision is slightly higher for the unfiltered case (being also the best result for the different transcript values for the unfiltered case of title terms). In this particular case, it could be that by considering only 10 features of the title, but 45000 for the transcript, the effect of the filtering of the title is diluted. However, this is not a very significant difference. On the other hand, as previously observed, the best results, both in terms of precision and recall, are obtained with 35000 transcript features in the case of filtering the title terms.

Hybrid weights setting. In Figure 3 we make a comparison of precision and recall of two different sets of users, using different weights for the recommender modules of our hybrid LRS. We define a first set of users of which we have little knowledge as those who have only seen 1 to 9 videos in the data set (815 users), calling them new users. The second set are the regular users previously used, formed by users that have already seen between 10 and 150 videos in the data 


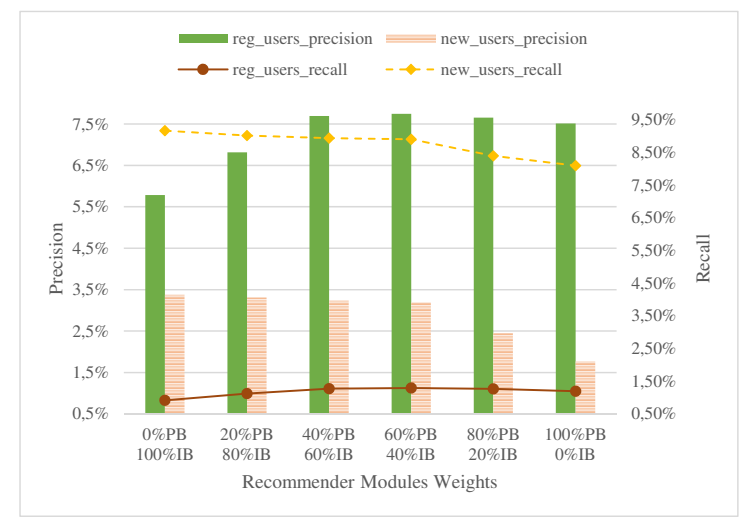

Fig. 3: Precision and recall comparison of regular users and new users, using different LRS modules weights.

set (1044 users). For all regular users, the best precision and recall is obtained with balanced weights, i.e., $\left\{w_{P B}=40 \% ; w_{I B}=60 \%\right\}$ and $\left\{w_{P B}=60 \% ; w_{I B}=\right.$ $40 \%\}$. However, for new users, the best precision and recall values are obtained with low $w_{P B}$, with $15.83 \%$ of new users receiving successful recommendations.

Finally, as there is not a previous recommender module in mediaUPV to compare with, we also calculated random recommendations for new and regular users, taking the highest value of 50 different random simulations (since random recommendation obtains 0 successful recommendations in most cases). Thus, a precision of $0.0156 \%$ and recall $0.0389 \%$ was achieved for regular users, and a precision of $0.0507 \%$ and recall $0.0167 \%$ for new users.

Discussion. For new users, the item-based module provides better results because low information about their activity is available, so the profile module is not able to offer good recommendations. This is not the case for regular users, since there is enough information about their profiles and therefore, a balanced weight distribution between both modules gives the best results.

Furthermore, we emphasize that even though our hybrid LRS obtains a precision of $7.7 \%$ simulating a real environment, $24.33 \%$ regular users and $15.83 \%$ of new users received some good recommendation. In addition, the precision and recall obtained are significantly better than a random recommendation.

In conclusion, after this analysis we established the parameters of the LRS to be applied in production in the mediaUPV portal as 35000 transcript features, 0 title features, $w_{P B}=60 \%$ and $w_{I B}=40 \%$.

\subsection{Production Results}

In this section we show the results of the application of the LRS proposed to the mediaUPV portal. It should be noted that mediaUPV did not have a LRS until the application in October 2019 of the one proposed in this work. 
In Figure 4a, we show the number of accesses to the videos of the mediaUPV platform during the year 2019, in which our LRS was applied from October. As can be seen, the existence of the LRS has increased slightly the number of accesses to the videos by the users.

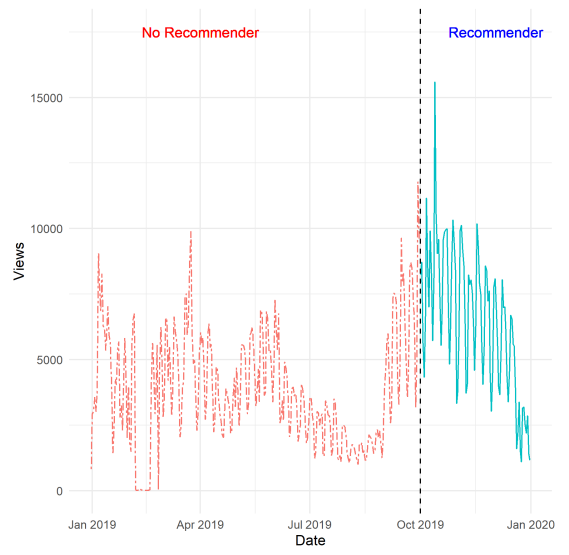

(a) Video access with and without recommender.

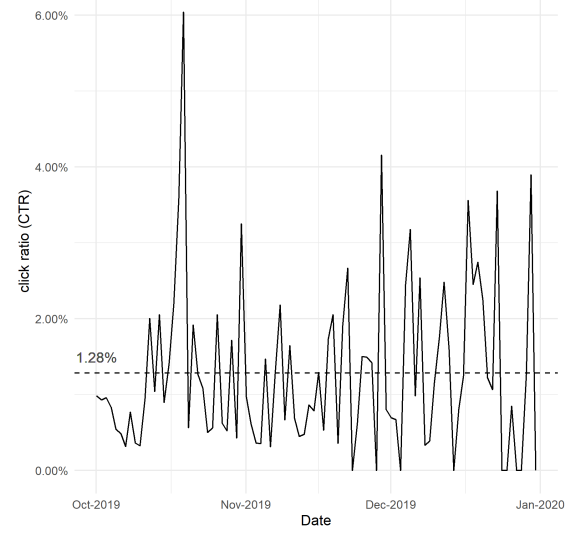

(b) Clicks in recommender (CTR).

Fig. 4: Performance measures in production.

A common way in industry to measure relative quality of a recommender system is the Click-Through Rate $(\mathrm{CTR}))^{9}$, that measures the percentage of clicks in the recommender per number of views. As the CTR is used by the ads industry, there is an ongoing interest in CTR prediction techniques [13. In the case of a generic recommender system, anything above $0.35 \%$ means you are doing a good job ${ }^{10}$. As can be seen in Figure $4 \mathrm{~b}$ the CTR is $1.28 \%$ on average, with notable peaks of around $4 \%$. These results can be considered quite satisfactory, since they imply that the recommendations made to users generate interest in them.

Finally, we point out the percentage of clicks on each of the recommended videos according to their order in the list, demonstrating the relevance of this order to users. Concretely, about $33 \%$ of users click on the first video, and more than half of the users click on the first two recommendations with a distribution that seems to be heavy tailed. In short, the most relevant video by far for users is the first one and then the rest of the videos follow a decreasing order of importance, which also points to a reasonable work of the presented LRS.

9 https://en.wikipedia.org/wiki/Click-throughrate

10 WWw.acquisio.com/blog/agency/what-is-a-good-click-through-rate-ctr/ 


\section{Conclusions}

This work proposes a new hybrid LRS based on content-based techniques capable of recommend learning videos based on viewing history and current video content. Thus, the LRS proposed is able to recommend not only to authenticated users but also to anonymous users from the mediaUPV portal, independently if they are teachers or students.

The hybrid LRS has been applied to a simulated environment, using a data set of learning videos and user profiles from the 2018-2019 academic year at UPV. The best hybrid LRS configuration obtained a $7.7 \%$ of precision and a $1.29 \%$ of recall, where a $24.33 \%$ of regular users received some useful recommendation.

Furthermore, this new approach has been applied in a real scenario, the mediaUPV portal of the UPV. This portal is mainly used by learners and trainers to access to useful LOs for their MOOCs and flipped classrooms. We can state that the application of this hybrid LRS to the mediaUPV portal was positive as it brought an increase in visits to the videos and had a significant CTR of $1.28 \%$ on average, with notable peaks of around $4 \%$.

As future work, we want to add a new module based on collaborative filtering, which provides recommendations based on viewing history of users similar to the current one, in order to increase the serendipity of the recommendations. In addition, it would be interesting to evaluate whether to add the classification of the mediaUPV videos obtained by [17] to the current characterization of the videos used by our proposal, which is currently based only on the video transcript.

\section{Acknowledgments}

This work was partially supported by MINECO/FEDER RTI2018-095390-BC31 and TIN2017-89156-R projects of the Spanish government, and PROMETEO/2018/002 project of Generalitat Valenciana. J. Jordán and V. Botti are funded by UPV PAID-06-18 project. J. Jordán is also funded by grant APOSTD/2018/010 of Generalitat Valenciana - Fondo Social Europeo.

\section{References}

1. Blei, D.M., Ng, A.Y., Jordan, M.I.: Latent dirichlet allocation. J. Mach. Learn. Res. 3, 993-1022 (Mar 2003)

2. Bobadilla, J., Serradilla, F., Hernando, A.: Collaborative filtering adapted to recommender systems of e-learning. Knowledge-Based Systems 22(4), 261 - 265 (2009)

3. Burke, R.: Hybrid recommender systems: Survey and experiments. User Modeling and User-Adapted Interaction 12(4), 331-370 (2002)

4. Chen, W., Niu, Z., Zhao, X., Li, Y.: A hybrid recommendation algorithm adapted in e-learning environments. World Wide Web 17(2), 271-284 (Mar 2014) 
5. van Dijck, J., Poell, T.: Higher Education in a Networked World: European Responses to U.S. MOOCs. International Journal of Communication: IJoC 9, 2674$2692(2015)$

6. Dwivedi, P., Bharadwaj, K.K.: e-learning recommender system for a group of learners based on the unified learner profile approach. Expert Systems 32(2), 264-276 (2015)

7. Herlocker, J., Konstan, J., Terveen, L., Riedl, J.: Evaluating collaborative filtering recommender systems. ACM Trans. Inf. Syst. 22(1), 5-53 (2004)

8. Institute and Committee of Electrical and Electronics Engineers. Learning Technology Standards: IEEE Standard for Learning Object Metadata. IEEE Standard 1484.12.1 (2002)

9. Klašnja-Milićević, A., Ivanović, M., Nanopoulos, A.: Recommender systems in elearning environments: a survey of the state-of-the-art and possible extensions. Artificial Intelligence Review 44(4), 571-604 (2015)

10. Maassen, P., Nerland, M., Yates, L. (eds.): Reconfiguring Knowledge in Higher Education. Higher Education Dynamics, 50, Springer International Publishing (2018)

11. MLLP research group (Universitat Politècnica de València): Tlp: The translecturesupv platform, http://www.mllp.upv.es/tlp

12. O'Flaherty, J., Phillips, C.: The use of flipped classrooms in higher education: A scoping review. The Internet and Higher Education 25, 85-95 (02 2015)

13. Richardson, M., Dominowska, E., Ragno, R.: Predicting clicks: estimating the clickthrough rate for new ads. In: Proceedings of the 16th international conference on World Wide Web. pp. 521-530 (2007)

14. Rodríguez, P., Heras, S., Palanca, J., Duque, N., Julián, V.: Argumentation-based hybrid recommender system for recommending learning objects. In: Multi-Agent Systems and Agreement Technologies. pp. 234-248. Springer International Publishing (2016)

15. Roehl, A., Reddy, S.L., Shannon, G.J.: The flipped classroom: an opportunity to engage millennial students through active learning strategies. Journal of Family and Consumer Sciences 105, 44-49 (2013)

16. Salton, G., Buckley, C.: Term-weighting approaches in automatic text retrieval. Information Processing \& Management 24(5), 513 - 523 (1988)

17. Stoica, A.S., Heras, S., Palanca, J., Julian, V., Mihaescu, M.C.: A semi-supervised method to classify educational videos. In: Hybrid Artificial Intelligent Systems. pp. 218-228. Springer International Publishing (2019)

18. Tarus, J.K., Niu, Z., Yousif, A.: A hybrid knowledge-based recommender system for e-learning based on ontology and sequential pattern mining. Future Generation Computer Systems 72, $37-48$ (2017)

19. Tucker, B.: The flipped classroom. online instruction at home frees class time for learning. Education Next Winter 2012, 82-83 (2012)

20. Turcu, G., Heras, S., Palanca, J., Julian, V., Mihaescu, M.C.: Towards a custom designed mechanism for indexing and retrieving video transcripts. In: Hybrid Artificial Intelligent Systems. pp. 299-309. Springer International Publishing (2019)

21. Turró, C., Morales, J.C., Busquets-Mataix, J.: A study on assessment results in a large scale flipped teaching experience. In: 4th International Conference on Higher Education Advances (HEAD'18). pp. 1039-1048 (2018)

22. Turró, C., Despujol, I., Busquets, J.: Networked teaching, the story of a success on creating e-learning content at Universitat Politècnica de València. EUNIS Journal of Higher Education (2014)

23. Zajda, Joseph;Rust, V.: Globalisation and Higher Education Reforms, vol. 15. Springer, Cham (2016) 